\title{
Measurement Results of Frequency Offset in DVB-C2 Receiver
}

\author{
Jae-Ho Lee*, Dong-Joon Choi*, Nam-Ho Hur*, Whan-Woo Kim** \\ * Smart Cable Broadcasting Research Division, ETRI, South Korea \\ ** Chungnam National University, South Korea \\ jaeholee@etri.re.kr, djchoi@etri.re.kr, namho@etri.re.kr, wwkim@cnu.ac.kr
}

\begin{abstract}
In this paper, we show the measurement results of frequency offset (FO) estimation in Digital Video Broadcasting for Cable version 2 (DVB-C2) receiver which uses orthogonal frequency-division multiplexing (OFDM). Because FO causes inter-carrier interference (ICI) in a multicarrier system, it should be estimated and compensated to improve the performance of a multicarrier receiver. FO can be divided into fractional frequency offset (FFO) and integer frequency offset (IFO) if FO is normalized to subcarrier spacing. The implemented FO estimator consists of FFO and IFO estimator. FFO estimator uses cyclic prefix (CP) in time domain and is implemented using coordinate rotation digital computer (CORDIC) algorithm. IFO estimator uses the correlation with unique synchronization sequence (USS) of preamble in frequency domain. First, we simulate the mean square error (MSE) of FO compensation algorithm w.r.t additive white Gaussian noise (AWGN) channel with computer simulation. Next, we implement FO estimator using in field programmable gate arrays (FPGAs). The implemented FO estimator has the resolution of $1 \mathrm{~Hz}$ approximately from measurement results of it.
\end{abstract}

Keywords-Frequency offset, CORDIC, unique synchronization sequence, FPGA

\section{INTRODUCTION}

FO is caused by an oscillator of transceiver and dependent on the accuracy of an oscillator. In single carrier system, received QAM symbols are rotated due to $\mathrm{FO}$, However, in multicarrier system such as DVB-C2 using OFDM, FO causes ICI among subcarriers and degrades the performance of a receiver [1]. Because $\mathrm{FO}$ of multicarrier system is more serious than single carrier system, it is preferable for FO estimator to have a fine resolution.

When FO is normalized to subcarrier spacing in multicarrier system, it consists of IFO and FFO. FFO estimator using $\mathrm{CP}$ in time domain can extract only the FFO of FO. Thus, IFO is estimated in frequency domain using USS after FFT.

We simulate the MSE of the FO estimation algorithm under AWGN with computer simulation and implement it with FPGAs. In addition, we measure the implemented FO estimator.

\section{FOC}

First, we introduce the implemented of OFDM system. Next, we explain the algorithm and simulated MSE of FO estimation. Last, we show the results of hardware measurement.

\section{A. OFDM System}

Figure 1 shows the OFDM system we implement.

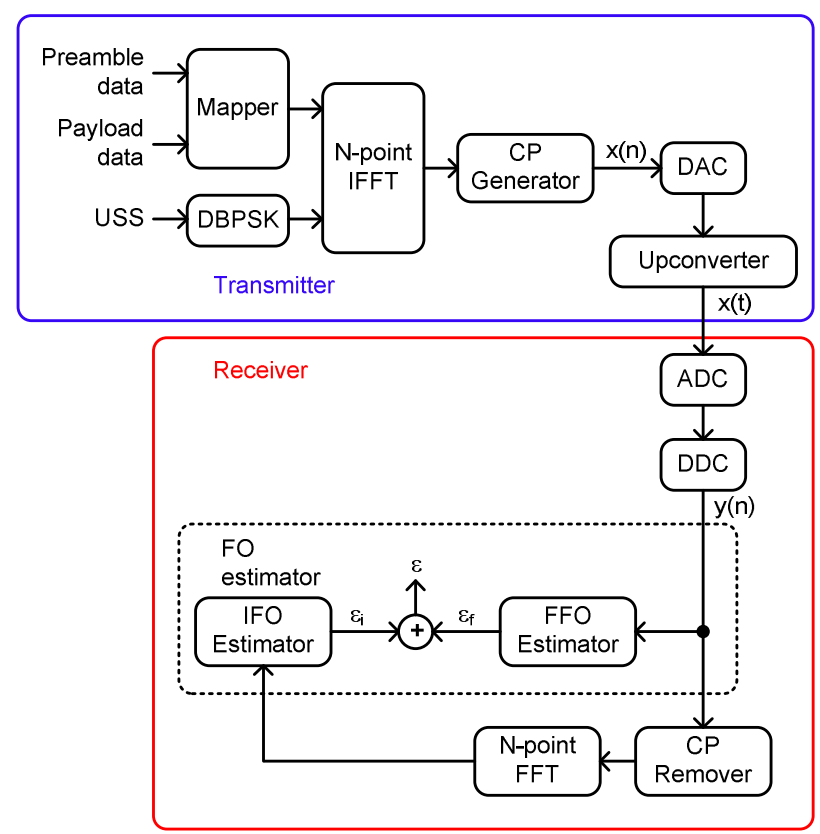

Figure 1. The block diagram of the implemented OFDM tranceiver

As shown in Figure 1, the transmitter in OFDM system consists of mapper, IFFT, CP generator, digital-to-analog converter (DAC) and upconverter. Mapper converts preamble and payload data into various QAM symbols by. USS is generated by two pseudo random binary sequences (PRBS) and is mapped by differential binary phase shift keying (DPBSK) for preamble pilot symbols. Preamble pilot symbols can be used for channel compensation and inserted for every 6 subcarriers [2], [3].

CP generator copies the last L samples of IFFT output and appends them to it. Upconverter modulates a baseband signal to $44 \mathrm{MHz}$ intermediate frequency (IF).

The receiver shown in Figure 1 consists of analog-to-digital converter (ADC), DDC, FOC, CP remover and FFT. DDC demodulates IF signal to baseband signal digitally. FOC is 
made of FFO estimator and IFO estimator. $\epsilon$ is normalized FO, $\epsilon_{f}$ is FFO and $\epsilon_{i}$ is IFO

Table 1 shows the system parameters for $6 \mathrm{MHz}$ channel bandwidth [2], [3].

TABLE 1. SYSTEM PARAMETERS OF OFDM TRANSMITTER

\begin{tabular}{|c|l|}
\hline $\begin{array}{c}\text { Number of total subcarriers } \\
\text { per OFDM symbol, } \mathrm{N}\end{array}$ & 4096 \\
\hline $\begin{array}{c}\text { Number of used subcarriers } \\
\text { per OFDM symbol, } \mathrm{N}_{\mathrm{USED}}\end{array}$ & 3409 \\
\hline Number of CP, $\mathrm{L}$ & 64 samples \\
\hline $\begin{array}{c}\text { Number of pilot subcarriers per OFDM } \\
\text { symbol in preamble, } \mathrm{N}_{\mathrm{PRP}}\end{array}$ & 569 \\
\hline Subcarrier spacing, $\Delta \mathrm{f}$ & $1.674 \mathrm{kHz}$ \\
\hline DAC and ADC bit resolution & $16 \mathrm{bits}$ \\
\hline IFFT/FFT period, $\mathrm{T}_{\mathrm{FFT}}$ & $593.7 \mu \mathrm{s}$ \\
\hline Sampling frequency, $\mathrm{f}_{\mathrm{s}}$ & $6.85 \mathrm{MHz}$ \\
\hline Tx IF frequency, $\mathrm{f}_{\mathrm{c} \_\mathrm{t}}$ & $44 \mathrm{MHz}$ \\
\hline DDC IF frequency, $\mathrm{f}_{\mathrm{c}}$ & $10.85 \mathrm{MHz}$ \\
\hline
\end{tabular}

\section{B. $D D C$}

Figure 2 shows the block diagram of DDC which consists of NCO, low pass filter (LPF) and Decimator.

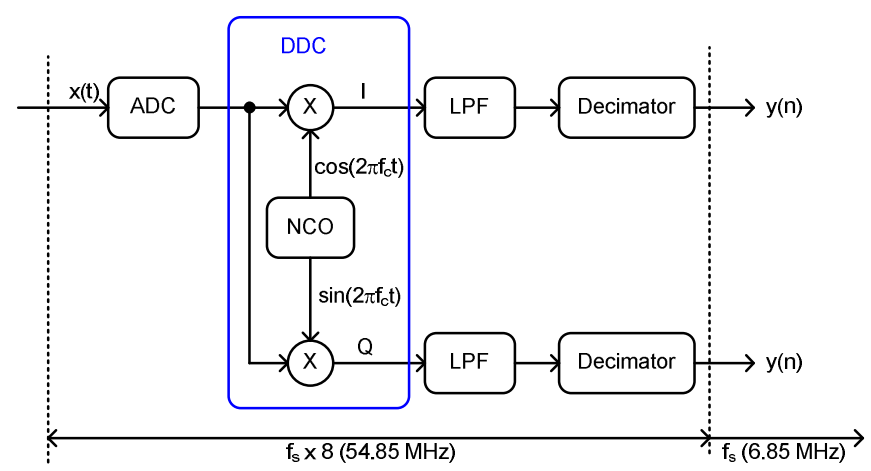

Figure 2. The block diagram of DDC

As shown in Figure 2, from ADC to decimator, they operate at eight times the sampling frequency. The $3 \mathrm{~dB}$ frequency of LPF is $3.42 \mathrm{MHz}$. The signal spectrum after ADC is shown in Figure 3.

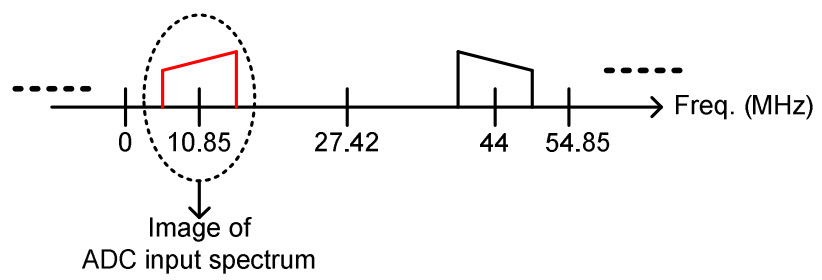

Figure 3. The spectrum after ADC

As shown in Figure 3, the image of ADC input spectrum, indicated by dotted line, for downconversion of input signal is used. In other words, $\sin \left(2 \pi f_{c} t\right)$ is used instead of $-\sin \left(2 \pi f_{c} t\right)$ shown in Figure 2. The factor of decimator is 8 .

\section{Estimation of FFO and IFO}

FO can be normalized to $\Delta \mathrm{f}$ and is expressed as equation (1).

$$
\frac{F O}{\Delta f}=\varepsilon=\epsilon_{f}+\epsilon_{i}
$$

where $\epsilon$ is normalized FO, $\epsilon_{f}$ is FFO and $\epsilon_{i}$ is IFO.

Figure 4 shows FFO estimator [4], [5] , [6], [7], [8], [9], [10].

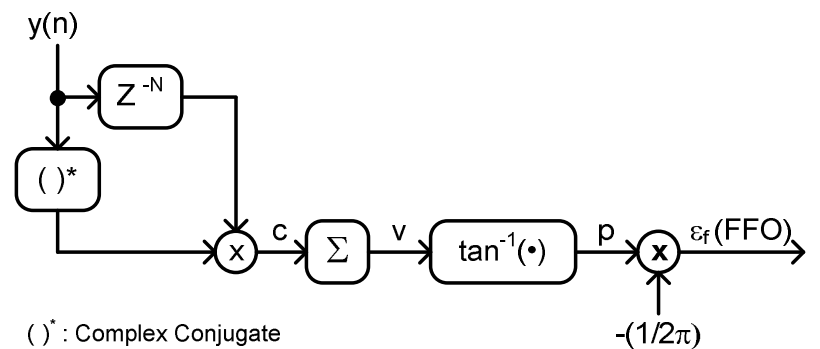

Figure 4. FFO estiamtor

The estimation of $\epsilon_{f}$ in Figure 4 can be expressed as equation (2) and estimated in time domain using CP of OFDM symbol.

$$
\begin{aligned}
\mathrm{y}(\mathrm{n}) & =x(n) e^{\frac{j 2 \pi \epsilon n}{N}}+z(n) \\
c(n) & =y^{*}(n) y(n-N) \\
& =x^{*}(n) x(n-N) e^{\frac{-j 2 \pi \epsilon n}{N}} e^{\frac{j 2 \pi \epsilon(n-N)}{N}}+z^{\prime}(n) \\
& =|x(n)|^{2} e^{-j 2 \pi \epsilon}+z^{\prime}(n) \\
v(n) & =e^{-j 2 \pi \epsilon}\left(\frac{1}{L}\right) \sum_{n=0}^{L-1}|x(n)|^{2}+z^{\prime}(n) \\
& =\operatorname{Ae}^{-j 2 \pi \epsilon}+n \operatorname{noise} \\
-2 \pi \epsilon & =\tan ^{-1}\left\{\frac{\operatorname{Imag}(v)}{\operatorname{Real}(v)}\right\}< \pm \pi \rightarrow|\varepsilon|<0.5
\end{aligned}
$$

where $\mathrm{x}(\mathrm{n})$ is IFFT output, $\mathrm{z}(\mathrm{n})$ and $\mathrm{z}^{\prime}(\mathrm{n})$ are noise.

As shown in equation (2), the estimation of FO using CP can extract only the FFO $\left(\epsilon_{f}\right)$ of FO $(\epsilon)$ and is affected with CP length.

The function of $\tan ^{-1}(\quad)$ is implemented by CORDIC algorithm shown in Figure 5. 


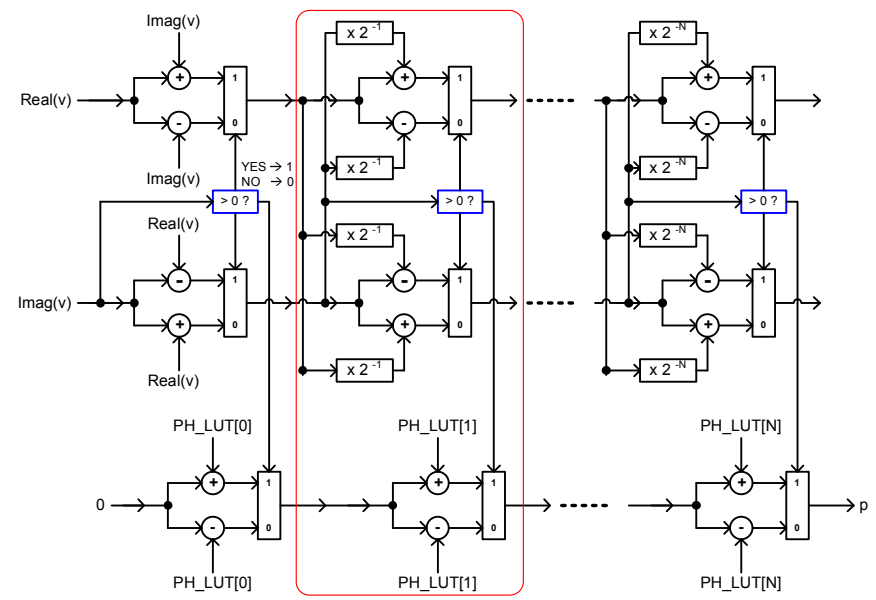

Figure 5. Implemented $\tan ^{-1}($ ) function

As shown in Figure 5, PH_LUT is a phase lookup table and generated in as equation (3).

$$
P H_{-} L U T[X]=\tan ^{-1}\left(2^{-X}\right) \text { radian } \quad X=0,1, \ldots, \mathrm{N}
$$

where $\mathrm{N}$ is the iteration number.

As $\mathrm{N}$ increases, the error of estimated phase (p) decreases. The red dotted line shown in Figure 5 is a basic cell. If $\mathrm{N}$ increases, the basic cell is added.

Figure 6 shows the estimated phase error when input (v) have a value of between $e^{-j \pi}$ and $e^{j \pi}$ and $\mathrm{N}$ is 16 .

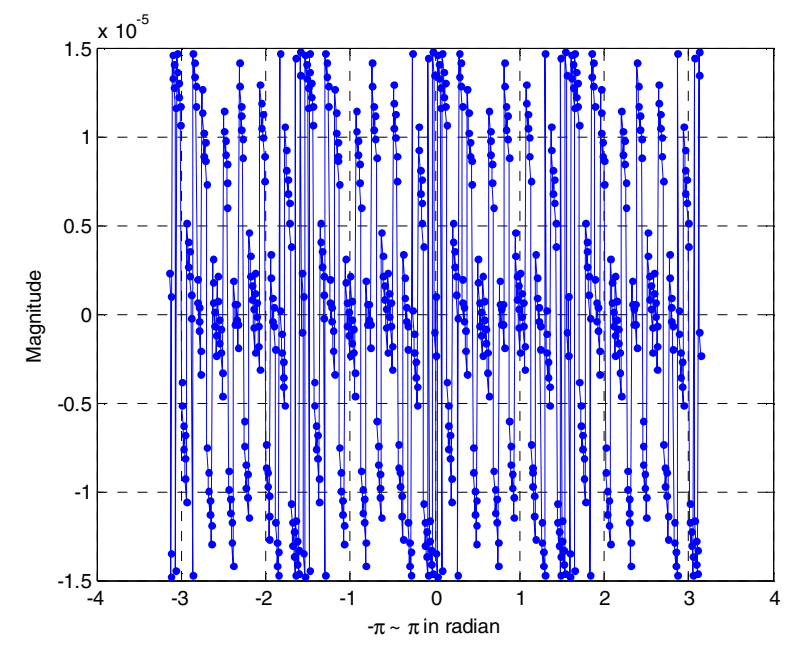

Figure 6. Estimated phase error when $\mathrm{N}$ is 16

As shown in Figure 6, the estimated phase error is very small. IFO $\left(\epsilon_{i}\right)$ of FO $(\epsilon)$ is estimated in frequency domain after FFT and IFO estimator is shown in Figure 7 [3].

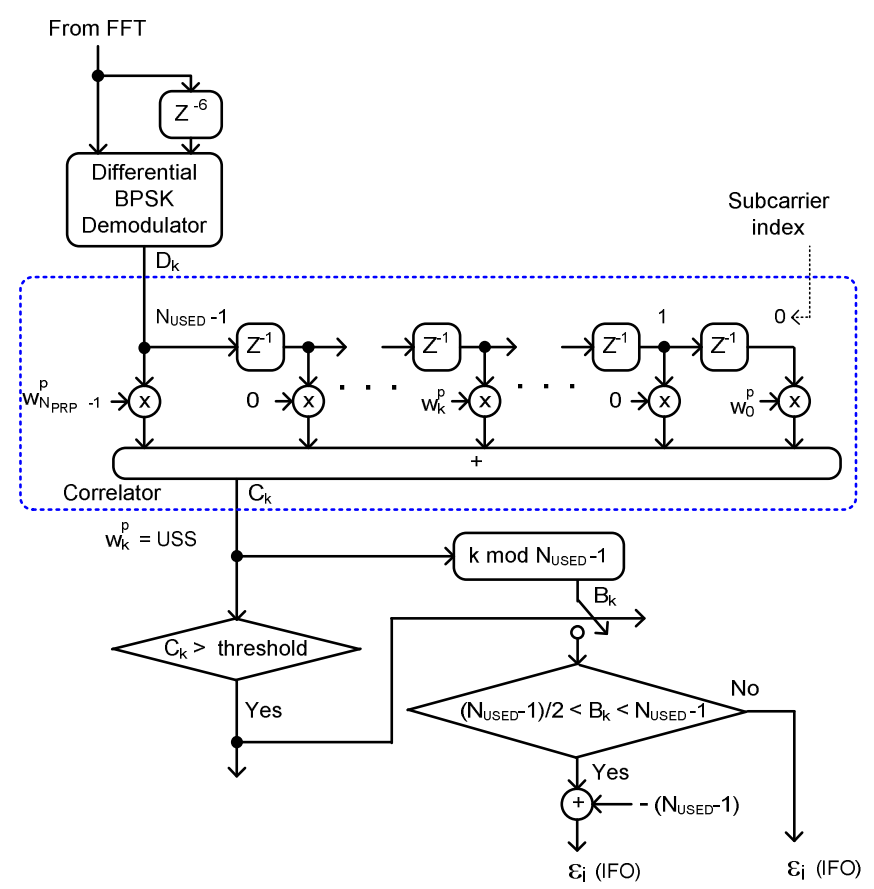

Figure 7. IFO estimator

As shown in Figure 7, $\mathrm{k}$ is the subcarrier index and IFO $\left(\epsilon_{i}\right)$ is estimated using USS and a correlator.

We simulate to evaluate the MSE of FO estimation under AWGN which can be expressed as equation (5).

$$
M S E=\frac{\sum_{M}\left|\epsilon_{\text {given }}-\epsilon_{\text {estimated }}\right|^{2}}{M}
$$

where $\mathrm{M}$ is the number of simulation iteration, $\epsilon_{\text {given }}$ is given frequency offset and $\epsilon_{\text {estimated }}$ is the estimated frequency offset.

Figure 10 shows the MSEs under AWGN.

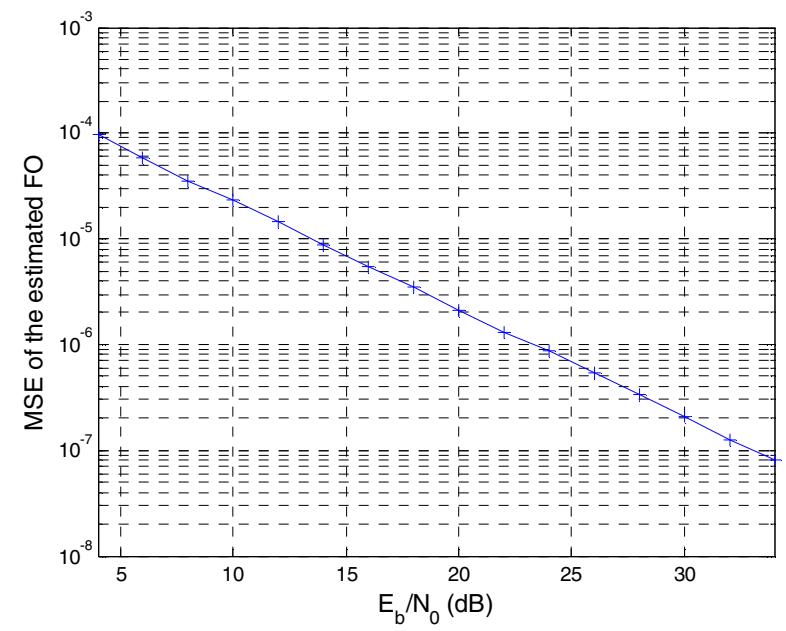

Figure 8. MSE of the estimated FO under AWGN when FO is $2.0925 \mathrm{kHz}$ 
As CP length and $\mathrm{E}_{\mathrm{b}} / \mathrm{N}_{0}$ increase, the MSEs decrease monotonically.

\section{III.Measurement Results}

Figure 9 shows the spectrum of $\mathrm{ADC}$ input whose center frequency is $44 \mathrm{MHz}$ and channel power is $-14.43 \mathrm{dBm} / 6 \mathrm{MHz}$.

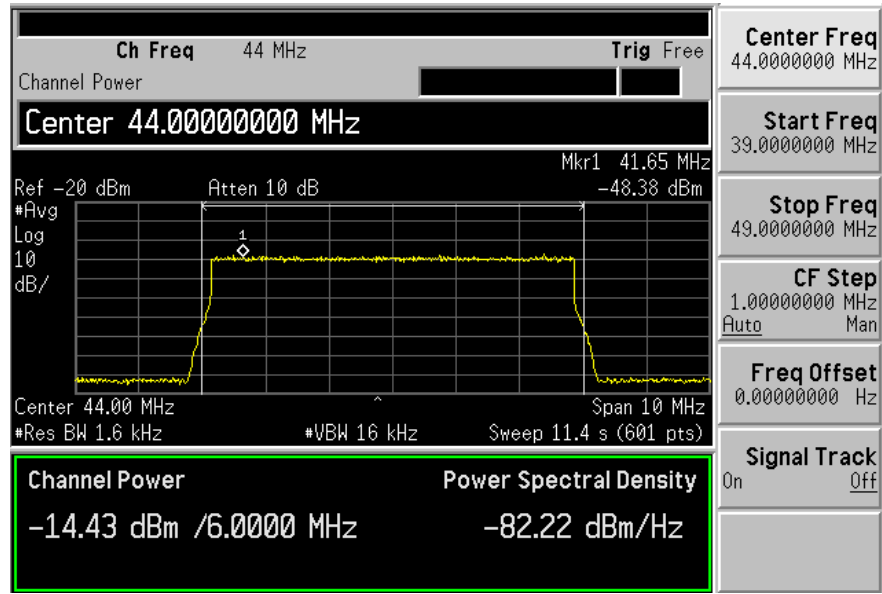

Figure 9. The spectrum of $\mathrm{ADC}$ input

Figure 10 shows the spectrum of DDC output and DDC + LPF output when the IF center frequency $\left(f_{c}\right)$ of DDC is $10.85 \mathrm{MHz}$.

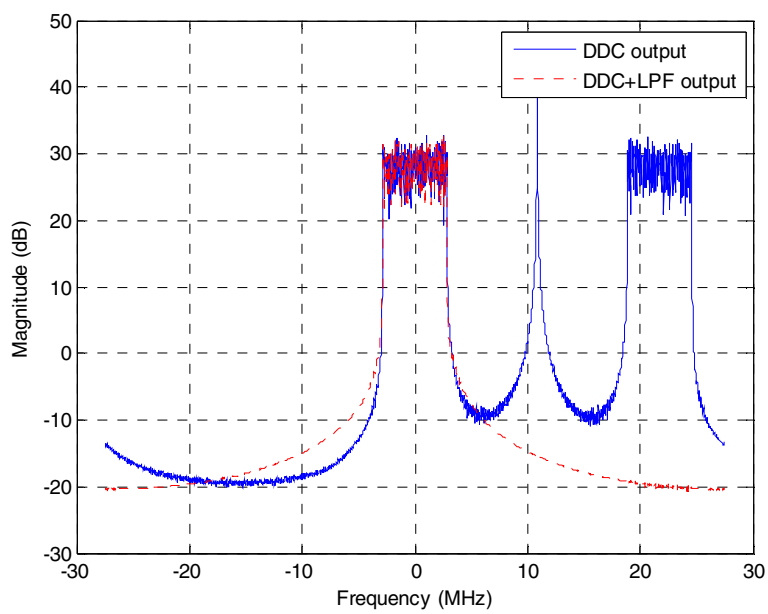

Figure 10. The spectrum of DDC and DDC+LPF output

As shown in Figure 10, the spectral line corresponds to the IF center frequency of DDC and is removed by LPF.

The estimated FO (nco_phi) from FFO and IFO estimator is shown in Figure 11.

国 $\$$ dmd_cmod:dmd_cmod|nco_phi

dmd_cmod.dmd_cmod|nco_sign

00149BA2h

Figure 11. The measurement of the estimated FO when IF center frequency is $10.85 \mathrm{MHz}$
If the nco_sign is 0 , it means positive FO. Otherwise, it represents negative FO. The nco_phi is eight-digit hexadecimal and the first three digit of it is the normalized IFO and the other 5 digit of it is the normalized FFO.

IFO has the bit resolution of $[12,12, \mathrm{u}]$ and the first 12 means the number of the total bits, and the second 12 expresses the number of the integer bits, and $\mathrm{u}$ represents unsigned number. Because the IFO is 001 , the floating value of it is 1 . FFO has the bit resolution of $[20,0, u]$. Because the FFO is 49BA2, the floating value of it is 0.286875 . Therefore, the un-normalized FO is $2.1568 \mathrm{kHz}(\Delta \mathrm{f} \times 1+\Delta \mathrm{f} \times 0.286875)$.

To test FO estimation, we change the IF center frequency intentionally listed in Table 2 .

TABLE 2. SEVERAL IF CENTER FREQEUNCY

\begin{tabular}{|c|l|}
\hline $\begin{array}{c}\text { IF center } \\
\text { frequency }\end{array}$ & Value \\
\hline $\mathrm{f}_{\mathrm{c}}$ & $10.85 \mathrm{MHz}+(\Delta \mathrm{f} \times 1)+(\Delta \mathrm{f} \times 0.286875)=$ \\
& $10.85 \mathrm{MHz}+2.1568 \mathrm{kHz}$ \\
\hline \multirow{2}{*}{$\mathrm{f}_{\mathrm{c}}$} & $10.85 \mathrm{MHz}+(\Delta \mathrm{f} \times 2)+(\Delta \mathrm{f} \times 0.286875)=$ \\
& $10.85 \mathrm{MHz}+3.8308 \mathrm{kHz}$ \\
\hline \multirow{2}{*}{$\mathrm{f}_{\mathrm{c}}$} & $10.85 \mathrm{MHz}+(\Delta \mathrm{f} \times 3)+(\Delta \mathrm{f} \times 0.286875)=$ \\
& $10.85 \mathrm{MHz}+5.5022 \mathrm{kHz}$ \\
\hline
\end{tabular}

Figure 12 shows the measurement results for three IF center frequency.

\begin{tabular}{|c|c|c|}
\hline$\Rightarrow$ & | dmd_cmod.dmd_cmod|nco_phi & 000004BAh \\
\hline$\Leftrightarrow$ & dmd_cmod:dmd_cmod|nco_sign & \\
\hline
\end{tabular}

(a)

\begin{tabular}{l|l|l} 
(2) & dmd_cmod:dmd_cmod|nco_sign & $0010048 \mathrm{Fh}$ \\
\hline
\end{tabular}

(b)

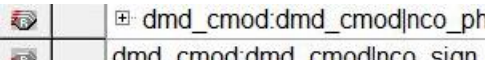
$00200441 \mathrm{~h}$

(c)

Figure 12. The measured FO (a) when IF center frequency is $10.85 \mathrm{MHz}+$ $2.1568 \mathrm{kHz}$ (b) Estimated FO when IF center frequency is $10.85 \mathrm{MHz}+$ $3.8308 \mathrm{kHz}$ (c) when IF center frequency is $10.85 \mathrm{MHz}+5.5022 \mathrm{kHz}$

As shown in Figure 12, Table 3 summarizes the measured FO with respect to various IF center frequency.

TABLE 3. IF FREQEUNCY

\begin{tabular}{|c|c|c|}
\hline $\begin{array}{c}\text { IF center } \\
\text { frequency }\end{array}$ & $\begin{array}{c}\text { FO } \\
\text { polarity }\end{array}$ & \multicolumn{1}{c|}{ Measured FO } \\
\hline $10.85 \mathrm{MHz}$ & positive & $2.1586 \mathrm{kHz}$ \\
\hline $10.85 \mathrm{MHz}+2.1568 \mathrm{kHz}$ & negative & $1.1539 \times 10^{-3} \mathrm{kHz}$ \\
\hline $10.85 \mathrm{MHz}+3.8308 \mathrm{kHz}$ & negative & $\left(1.674+1.1129 \times 10^{-3}\right) \mathrm{kHz}$ \\
\hline $10.85 \mathrm{MHz}+5.5022 \mathrm{kHz}$ & negative & $\left(3.348+1.0385 \times 10^{-3}\right) \mathrm{kHz}$ \\
\hline
\end{tabular}

As shown in Table 3, the implemented FO estimator has 1 $\mathrm{Hz}$ frequency resolution approximately.

\section{IV.CONCLUSION}


We implement FO estimator which consists of FFO and IFO estimator. The FFO estimator uses $\mathrm{CP}$ in time domain and the CORDIC algorithm is used. The IFO estimator uses the correlaiton with USS in frequency domain. The implemented FO estimator has $1 \mathrm{~Hz}$ resolution approximately from measurement results.

\section{ACKNOWLEDGMENT}

This work was supported by the Broadcast and Telecommunications R\&D program. [10-921-02-001, Development of Next-Generation Digital Cable Transmission Technology].

\section{REFERENCES}

[1] Yong Soo Cho, Jaekwon Kim, Won Young Yang and Chung G. Kang, MIMO-OFDM WIRELESS COMMUNICATIONS WITH MATLAB, John Wiley \& Sons (Asia) Pte Ltd, 2010.

[2] Digital Video Broadcasting (DVB); Frame structure channel coding and modulation for a second generation digital transmission system for cable systems (DVB-C2), DVB Document A138 April 2009

[3] Digital Video Broadcasting (DVB); Implementation Guidelines for second generation digital cable transmission system (DVB-C2), DVB Document A147, March 2010

[4] W. D. Warner and C. Leung, "OFDM/FM frame synchronization for mobile radio data communication," IEEE Trans. Veh. Technol., vol. 42, pp. 302-313, Aug. 1993.

[5] J. J. van de Beek, M. Sandell, M. Isaksson, and P. O. Börjesson, "Low complex frame synchronization in OFDM systems," in Proc. IEEE Int. Conf. Universal Personal Commun., Nov. 1995, pp. 982-986.

[6] Ming Lei, Minjian Zhao, Jie Zhong, and Yunlong Cai, "ML-Based Estimation Algorithm of Frequency Offset for $2 \times 2$ STBC-OFDM Systems", ETRI Journal. June. 2012, Vol. 34, No. 3, pp. 393-398.

[7] P.H. Moose, "A Technique for Orthogonal Frequency Division Multiplexing Frequency Offset Correction," IEEE Trans. Commun., vol. 42, no. 10, Oct. 1994, pp. 2908-2914.

[8] M. Morelli and U. Mengali, "An Improved Frequency Offset Estimator for OFDM Applications," IEEE Commun. Lett., vol. 3, no. 3, Mar. 1999, pp. 75-77.

[9] Ki-Doo Kim, Jung-Hun Oh, and Laurence B. Milstein, "The Performance Improvement of a Multicarrier DS-CDMA System Using both Time-Diversity and Frequency Offset", ETRI Journal. December. 1999, Vol. 21, No. 4, pp. 29-40.

[10] Hyoungsoo Lim and Dong Seung Kwon, "A Simple Scheme for Jitter Reduction in Phase-Differential Carrier Frequency Recovery Loop", ETRI Journal. June. 2006, Vol. 28, No. 2, pp. 275-281.

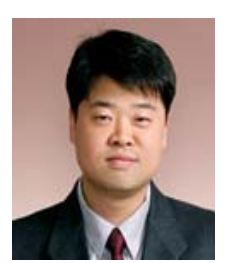

First A. Author Jae-Ho Lee was born in Namwon, Korea, in 1972. He received the B.S. and M.S. degree in electronics engineering from the Chonbuk National University, Korea, in 1997 and 1999, respectively. He has enrolled in a doctoral course at Chungnam National University in 2010. He joined in ETRI (Electronics and Telecommunications Research Institute) in 1999. Since 1999, he has participated in developing IEEE 802.11a system and the related data services. His current interest includes cable broadcasting, and OFDM system.

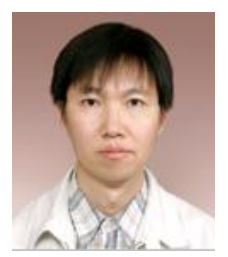

Dong-Joon Choi received the B.S. and M.S degree in electronics engineering from the Pohang University of science and technology, Korea, in 1991 and 1993, respectively. He joined in ETRI (Electronics and Telecommunication Research Institute) in 1993. Since 1993, he has participated in developing wireless MAC system and the related data services. His current interest includes cable broadcasting, DOCSIS, and

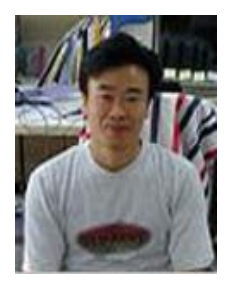

Namho Hur received the $\mathrm{BS}$, MS, and $\mathrm{PhD}$ degrees in electrical and electronic engineering from Pohang University of Science and Technology (POSTECH), Pohang, Korea, in 1992 1994, and 2000. He is currently with the Digital Broadcasting Research Division, Electronics and Telecommunications Research Institute (ETRI), Daejeon, Korea. As a research scientist, he spent a year with Communications Research Centre Canada (CRC) from 2003 to 2004. His main research interests are ac motor drives, control theory and its application to power electronics, high-performance power converter/inverter systems, three dimensional television (3DTV) broadcasting systems, and perceptual requirements of stereoscopic multiview video systems.

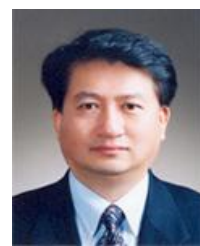

Whan-Woo Kim received the B.S. degree in electronics engineering from Seoul University, Korea, in 1977 and the M.S. and Ph.D. degrees from KAIST and University of Utah, Korea and USA in 1979 and 1988, respectively.

OFDM system 